\title{
Linear and nonlinear Zeno effects in an optical coupler
}

\author{
F. Kh. Abdullaev ${ }^{1}$, V. V. Konotop ${ }^{1,2}$, and V. S. Shchesnovich ${ }^{3}$ \\ ${ }^{1}$ Centro de Física Teórica e Computacional, Faculdade de Ciências, \\ Universidade de Lisboa, Avenida Professor Gama Pinto 2, Lisboa 1649-003, \\ Portugal ${ }^{2}$ Departamento de Física, Faculdade de Ciências, Universidade de Lisboa, \\ Campo Grande, Ed. C8, Piso 6, Lisboa 1749-016, Portugal \\ ${ }^{3}$ Centro de Ciências Naturais e Humanas, Universidade Federal do ABC, Santo André, SP 09210-170, Brazil
}

\begin{abstract}
It is shown that in a simple coupler where one of the waveguides is subject to controlled losses of the electric field, it is possible to observe optical analog of the linear and nonlinear quantum Zeno effects. The phenomenon consists in a counter-intuitive enhancement of transparency of the coupler with the increase of the dissipation and represents an optical analogue of the quantum Zeno effect. Experimental realization of the phenomenon based on the use of the chalcogenide glasses is proposed. The system allows for observation of the cross-over between the linear and nonlinear Zeno effects, as well as effective manipulation of light transmission through the coupler.
\end{abstract}

\section{INTRODUCTION}

Decay of a quantum system, either because it is in a metastable state or due to its interaction with an external system (say, with a measuring apparatus), is one of the fundamental problems of the quantum mechanics. Already more than fifty years ago it was proven that the decay of a quantum metastable system is, in general, non-exponential [1, 2] (see also the reviews [3, 4]). Ten years later in Ref. [5] it was pointed that a quantum system undergoing frequent measurements does not decay at all in the limit of the infinitely-frequent measurements. This remarkable phenomenon was termed by the authors the quantum "Zeno's paradox". The Zeno's paradox, i.e. the total inhibition of the decay, requires, however, unrealistic conditions and shows only as the Zeno effect, i.e. the decrease of the decay rate by frequent observations, either pulsed or continuous. The Zeno effect was observed experimentally by studying the decay of continuously counted beryllium ions [6], the escape of cold atoms from an accelerating optical lattice [7], the control of spin motion by circularly polarized light [8], the decay of the externally driven mixture of two hyperfine states of rubidium atoms [9], and the production of cold molecular gases [10]. There is also the opposite effect, i.e. the acceleration of the decay by observations, termed the anti-Zeno effect, which is even more ubiquitous in the quantum systems [11].

It was argued that the quantum Zeno and anti-Zeno effects can be explained from the purely dynamical point of view, without any reference to the projection postulate of the quantum mechanics 12. In this respect, in Ref. [13, 14] it is shown that the Zeno effect can be understood within the framework of the mean field description, when the latter can be applied, thus providing the link between purely quantum and classical systems.

The importance of the Zeno effect goes beyond the quantum systems. An analogy between the quantum Zeno effect and the decay of light in an array of optical waveguides, was suggested in Ref. [15]. Namely, the authors found an exact solution which showed a non- exponential decay of the field in one of the waveguides. Modeling of the quantum Zeno effect in the limit of frequent measurements using down conversion of light in the sliced nonlinear crystal was considered in Ref. [16]. The effect has been mimicked by the wave process in a $\chi^{(2)}$ coupler with a linear and nonlinear arms, since in the strong coupling limit the pump photons propagate in the nonlinear arm without decay. The analogy between the inhibition of losses of molecules and the enhanced reflection of light from a medium with a very high absorption was also noticed in [9].

Meantime, in the mean field models explored in Refs. [13, 14] inter-atomic interactions play an important role, leading to the nonlinear terms in the resulting dynamical equations. In its turn, the nonlinearity introduces qualitative differences in the Zeno effect, in particular dramatically reducing the decay rate [14] compared to the case of noninteracting atoms. This phenomenon, the enhancement of the effect by the inter-atomic interactions, in Ref. 14] was termed the nonlinear Zeno effect (since, when the nonlinearity is negligible, it reduces to the usual linear Zeno effect).

Mathematically, the mean field description of a BoseEinstein condensate (BEC) and of the light propagation in Kerr-type media are known to have many similarities, due to the same (Gross-Pitaevskii or nonlinear Schrödinger) equation describing the both phenomena. Furthermore, the linear Zeno effect, is observable not only in pure quantum statement, but also in the meanfield approximation [14]. This immediately suggests that detecting the Zeno dynamics, is possible in the classical systems, and in particular, in the nonlinear optics, thus offering new possibilities for managing of light [17]. Namely, one can expect the counter-intuitive reduction of attenuation of the total field amplitude (which would correspond to the reduction of losses of atoms in the BEC case) by increasing the losses in some parts of the system (an analogy to the increasing of the removal rate of atoms in the case of BEC).

To report on a very basic system where analogs of the linear and nonlinear Zeno effects can be observed and exploited is the main goal of the present paper. More 
specifically, we explore the mathematical analogy of the semi-classical dynamics of a BEC in a double well potential subject to removal of atoms [14], with light propagation in a nonlinear optical coupler, in which one of the arms is subject to controllable losses.

The paper is organized as follows. First in Sec. II we consider two well known models of dissipative oscillators, which illustrate the classical analogues of the Zeno phenomenon (originally introduced in the quantum measurement theory). Next, in Sec. III we discuss possible experimental settings allowing observation of the phenomenon in optics. In Sec. [V] the theory of optical nonlinear Zeno effect is considered in details. Sec. $\mathrm{V}$ is devoted to comparative analysis of the linear and nonlinear Zeno effects. The outcomes are summarized in the Conclusion.

\section{TWO TRIVIAL EXAMPLES.}

Before going into details of the optical system, let us first give a simple insight on the pure classical origin of the phenomenon of inhibition of the field attenuation by strong dissipation. First, we recall the well-known fact, that increase of the dissipation $\alpha$ of an overdamped $(\alpha \gg$ $\omega)$ oscillator $\ddot{x}+\alpha \dot{x}+\omega^{2} x=0$, results in decrease of the attenuation of the oscillations. Indeed the decay rate $R \approx$ $\omega^{2} / \alpha$ approaches zero, when the dissipation coefficient $\alpha$ goes to infinity. But the amplitude of oscillations in this case is also nearly zero. However, the coupling of another linear oscillator to the dissipative one,

$$
\ddot{x}_{1}+\alpha \dot{x}_{1}+\omega^{2} x_{1}+\kappa x_{2}=0, \quad \ddot{x}_{2}+\omega^{2} x_{2}+\kappa x_{1}=0,
$$

allows one to observe the inhibition of attenuation due to strong dissipation by following a finite amplitude $x_{2}$. Indeed, the characteristic equation,

$$
\lambda=\frac{\lambda^{4}+2 \lambda^{2} \omega^{2}+\kappa^{2}-\omega^{4}}{\alpha\left(\lambda^{2}+\omega^{2}\right)},
$$

evidently has the small root $\lambda \approx\left(\kappa^{2}-\omega^{4}\right) /\left(\alpha \omega^{2}\right)$ which appears for $\alpha \gg \kappa^{2} / \omega^{2}-\omega^{2}>0$. Thus, one of the dynamical regimes of the system is characterized by the decay rate which goes to zero in the overdamped case, moreover the relation between the amplitudes of the damped and undamped oscillators reads $\left|x_{1} / x_{2}\right| \rightarrow \omega^{2} / \kappa<1$ as $\alpha \rightarrow \infty$. In other words, strong dissipation in one of the oscillators can attenuate the energy decay in the whole system. On the other hand, the last example illustrates that if the coupling is of the same order as the eigenfrequencies of the subsystems, the energy is distributed between the two subsystems in approximately equal parts. This does not allow for further decrease of the decay rate of the energy, because its large part is concentrated in the damped subsystem.

The phenomenon descried above for the linear oscillators can be viewed as a classical analog of the linear Zeno effect. The nonlinearity changes the situation dramatically. This case, however does not allow for complete analytical treatment, any more, and that is why we now turn to the specific nonlinear system, which will be studies numerically. We consider an optical coupler composed of two Kerr-type waveguides, one arm of which is subject to relatively strong field losses. We will show that such a coupler mimics the quantum Zeno effect, allowing one to follow, in a simple optical experiment, the crossover between the linear (weak intensities) and nonlinear (strong intensities) Zeno effects, thus providing a deep analogy between the effect of dissipation in the classical and quantum systems. In particular, we will also show that strong losses of the field in one of the waveguides can significantly enhance the transmittance of the coupler as a whole.

\section{THE COUPLER AND A POSSIBLE EXPERIMENTAL SETTING.}

The optical fields in the two tunnel-coupled nonlinear optical fibers [18] (alternatively, one can consider two linearly couple waveguides [19]) are described by the system

$$
\begin{aligned}
& -i \frac{d a_{1}}{d z}=\left(\beta_{1}+i \alpha_{1}\right) a_{1} \pm \gamma\left|a_{1}\right|^{2} a_{1}+\kappa a_{2}, \\
& -i \frac{d a_{2}}{d z}=\left(\beta_{2}+i \alpha_{2}\right) a_{2} \pm \gamma\left|a_{2}\right|^{2} a_{2}+\kappa a_{1} .
\end{aligned}
$$

Here $a_{1,2}$ are the properly normalized fields in each arm of the coupler, $\kappa$ is the coupling coefficient measuring the spatial overlap between the channels, the upper and lower signs correspond to the focusing $(+)$ and defocusing $(-)$ media, $\beta_{j}(j=1,2)$ are the modal propagation constants of the cores, $\gamma=2 \pi n_{2} / \lambda A_{e f f}, n_{2}$ is the Kerr nonlinearity parameter, $A_{e f f}$ is the effective cross section of the fiber, $\lambda$ is the wavelength, and the loss coefficient $\alpha_{j}>0$ stands for the field absorption in the $j$ th waveguide.

Our aim is to employ the manageable losses, i.e. a control over the coefficients $\alpha_{1,2}$, in order to observe different regimes of the light transmission through the coupler. Since in optics one cannot easily manipulate with $z$, i.e. with the length of the coupler, we are interested in realizing different dynamical regimes with a single given coupler (rather than using several couplers having different characteristics). This contrasts with the BEC case where the propagation variable $z$ corresponds to time (see e.g. 14]) and can be easily varied. For this reason the most suitable experimental setting could be with a coupler, whose properties strongly depend on the wavelength of the input beam (alternatively one can consider flexible change of the optical properties using temporal gradients, active doping, etc.).

An experimentally feasible realization of the described nonlinear directional coupler can be based on the use of the $\mathrm{As}_{2} \mathrm{Se}_{3}$ chalcogenide glass. For this material the intrinsic nonlinearity can be up to three orders of magnitude greater than that of the pure silica fibers [20 22]. More specifically, one can consider the material losses in the chalcogenide glasses, where the Kerr nonlinearity parameter is $n_{2}=1.1 \cdot 10^{-13} \mathrm{~cm}^{2} / \mathrm{W}$, that is 400 times of 
the nonlinearity of the fused silica fiber. However, what is even more important for our aims, is that the absorption rate of at least one of the coupler arms can be changed dramatically during the experiment. Say, in the mentioned chalcogenide glasse $\alpha$ can be on the order of a few $\mathrm{dB} / \mathrm{m}$ and is very sensitive to the wavelength. Thus, a practical control over the absorption can be performed by using the dependence of the loss coefficients $\alpha_{1,2}$ on the wavelength of the incident light.

To implement this idea, it is necessary to produce the two arms of the coupler using chalcogenide glasses of different types. In particular, one can consider the standard sulphide fiber in one arm of the coupler and the lowest loss sulphide fibers [23] in the other arm. Such sulphide fibers have a particularly narrow attenuation peak at the wavelength $\lambda_{0} \approx 3 \mu \mathrm{m}$. The behavior of the absorption coefficient $\alpha$ in the vicinity of this peak can be modeled by the Lorentzian curve (here we use the experimental results reported in [24]):

$$
\alpha_{1}(\lambda)=\alpha_{1,0}+\frac{\alpha_{1,1} \Gamma^{2}}{\left(\lambda-\lambda_{0}\right)^{2}+\Gamma^{2}},
$$

where $\Gamma \approx(0.5 \div 1) \mu \mathrm{m}$ and $\alpha_{1,0} \approx 0.5 \mathrm{~dB} / \mathrm{m}$ and $\alpha_{1,1} \sim$ $5 \mathrm{~dB} / \mathrm{m}$ for a usual sulfide fiber. Varying the wavelength about $\lambda_{0} \approx 3 \mu \mathrm{m}$ in the interval $\lambda_{0} \pm 0.5 \mu \mathrm{m}$ the loss can be varied in the standard sulfide fiber by $(0.5 \div 5) \mathrm{dB} / \mathrm{m}$, and in the lowest loss sulfide fiber in the interval $(0.05 \div$ $0.2) \mathrm{dB} / \mathrm{m}$. Even larger attenuation can be achieved for chalcogenide fibers $30 \mathrm{Ge}-10 \mathrm{As}-30 \mathrm{Se}-30 \mathrm{Te}$, where the pick attenuation is on the order of $(5 \div 30) \mathrm{dB} / \mathrm{m}$ observed for $\lambda \approx 4.5 \mu \mathrm{m}$.

\section{THE NONLINEAR OPTICAL ZENO EFFECT.}

Thus we consider the situation when one of the waveguides (waveguide 1) is subjected to controllable losses (as discussed above), while the second one (waveguide 2) is operating in the transparency regime, i.e. when $\alpha_{1} \gg \alpha_{2}$. Respectively, we simplify the problem setting in what follows $\alpha_{2}=0$.

We start with the estimate of the effective losses, designated below as $\widetilde{\alpha}_{2}$, in the transparent arm of the coupler, which occur due to the energy exchange between the arms. For $z \gg \alpha_{1}^{-1}$, one can adiabatically eliminate $a_{1}$ from the system (11). Moreover, assuming that $\alpha_{1} \gg \kappa$ we obtain: $\left|a_{1}\right|^{2} \approx \frac{\widetilde{\alpha}_{2}}{\alpha_{1}}\left|a_{2}\right|^{2} \ll\left|a_{2}\right|^{2}$ and

$$
-i \frac{d a_{2}}{d z} \approx\left(i \widetilde{\alpha}_{2}+\widetilde{\beta}_{2}+\widetilde{\gamma}\left|a_{2}\right|^{2}\right) a_{2} .
$$

Here $\widetilde{\beta}_{2}=\beta_{2}+\widetilde{\alpha}_{2}\left(\beta_{2}-\beta_{1}\right) / \alpha_{1}$ and $\widetilde{\gamma}=\gamma\left(1+\widetilde{\alpha}_{2} / \alpha_{1}\right)$, with the effective $z$-dependent attenuation rate:

$$
\widetilde{\alpha}_{2}=\frac{\alpha_{1} \kappa^{2}}{\left(\beta_{2}-\beta_{1}+\gamma\left|a_{2}\right|^{2}\right)^{2}+\alpha_{1}^{2}} .
$$

First of all, we observe that $\widetilde{\alpha}_{2}$ decays with increase of the difference $\beta_{2}-\beta_{1}$ or the nonlinearity (the term $\gamma\left|a_{2}\right|^{2}$ in the denominator). This behavior is natural because the difference in the propagation constants $\beta_{1,2}$ results in incomplete energy transfer between the arms, whereas the nonlinearity effectively acts as an additional amplitudedependent detuning. In practical terms, however, the effect due to the constant linear detuning is negligible, because $\beta_{2}-\beta_{1}$ is typically too small, whereas the nonlinearity can result in an appreciable effect. Thus, the effective attenuation rate $\widetilde{\alpha}_{2}$ decays either with increase of the absorption $\alpha_{1} \rightarrow \infty$ (the linear Zeno effect), or (for given losses $\alpha_{1}$ ) with the intensity of the light in the transparent arm, tending to zero in the formal limit $\left|a_{2}\right|^{2} \rightarrow \infty$ (the nonlinear Zeno effect).

Moreover, the anti-Zeno effect, i.e. the increase of the attenuation with the increase of the loss coefficient $\alpha_{1}$ can be observed merely due to the presence of a strong nonlinearity. Such an effect, however, is not counterintuitive in our setup. In fact, it is rather trivial: for $\gamma\left|a_{2}\right|^{2} \gg \alpha_{1}$ Eq. (4) tells us that the ratio $\left|a_{1}\right|^{2} /\left|a_{2}\right|^{2} \approx$ $\widetilde{\alpha}_{2} / \alpha_{1}$ is independent of $\alpha_{1}$, which means that, in the Zeno regime $z \gg \alpha_{1}^{-1}$, increasing the loss coefficient must increase the actual attenuation.

In order to perform the complete numerical study of the coupler we introduce the real amplitudes and phase of the fields: $a_{j}=\rho_{j} \exp \left(i \phi_{j}\right), j=1,2$, the relative difference in the energy flows in the two arms $F=\left(\left|a_{1}\right|^{2}-\right.$ $\left.\left|a_{2}\right|^{2}\right) /\left(\left|a_{1}\right|^{2}+\left|a_{2}\right|^{2}\right)$, the total energy flow in the coupler $Q=\left(\left|a_{1}\right|^{2}+\left|a_{2}\right|^{2}\right) / P_{0}$, normalized to the input flow $P_{0}=$ $\left|a_{10}\right|^{2}+\left|a_{20}\right|^{2}$, as well as the phase mismatch $\phi=\phi_{1}-\phi_{2}$. Then the original system (1) is reduced to

$$
\begin{aligned}
& F_{Z}=-g\left(1-F^{2}\right)+2 \sqrt{1-F^{2}} \sin (\phi), \\
& \phi_{Z}=\frac{\left(\beta_{1}-\beta_{2}\right)}{\kappa} \pm 4 \delta F Q-2 \frac{F}{\sqrt{1-F^{2}}} \cos (\phi), \\
& Q_{Z}=-g Q(1+F)
\end{aligned}
$$

where $g=\alpha_{1} / \kappa, \delta=P_{0} / P_{c}$, and the distance is normalized on the linear coupling length $L=1 / \kappa$, i.e. $Z=z / L=z \kappa$. Here we also introduced the critical power $P_{c}=4 \kappa / \gamma$, The critical power $P_{c}$ which separates the regimes with periodic exchange energy between the arms for $P<P_{c}$ and the localization of energy in one of the waveguides if $P>P_{c}$ [18. For a fiber based on the chalcogenide glass, described above, the critical power $P_{c} \sim 1 \mathrm{~W}$ and the coupling length $\mathrm{L}$ varies in the interval $(0.1 \div 1) \mathrm{m}$.

Notice that mathematically the system (5) coincides with the one describing a BEC in a double-well trap subject to elimination of atoms from one of the wells [14]. The coupler mimics the nonlinear Zeno effect in a BEC in a double-well trap, where the time is replaced by the propagation distance in the coupler and the electric fields in the arms of the coupler correspond to the number of quantum particles in the potential wells. The system (5) also resembles the evolution of Bose-Hubbard dimer with non-Hermitian Hamiltonian [25] 


\section{LINEAR $V S$ NONLINEAR ZENO EFFECTS.}

Passing to the numerical study of the system (5) we estimate that for the length $L$ on the order of one meter the value of the absorption coefficient $g$ can be changed in chalcogenide fibers by up to 20 times. In the empiric formula (2) the values of the dimensionless parameters are as follows: $g_{1,0}=\alpha_{1,0} / \kappa \sim 1$ and $g_{1,1} \alpha_{1,1} / \kappa \sim(10 \div$ $20)$, while $\delta=P_{0} / P_{c}$ is in the interval $(0 \div 2.5)$.

Our main results are summarized in Fig. 1 In panels (a) and (b), where we show the dependence of the output signal vs the coupler length, the three different regimes are evident. At small distances from the coupler input, $Z \lesssim 0.2$, the standard exponential decay occurs. This stage does not depend significantly on the intensity of the input pulse (i.e. on $\delta$ in our notations). However at larger distances, $0.2 \lesssim Z \lesssim 2$, the system clearly reveals power-like decay. The power of the decay, however appears to be sensitive to the magnitude of the input power, i.e. to the nonlinearity of the system. The decay is much stronger at lower powers $(\delta \approx 0)$, corresponding to the linear Zeno effect, and much weaker for the input intensities above the critical value $(\delta=2)$, and may be termed the nonlinear Zeno effect. In all the cases the output beam is concentrated in the fiber/waveguide without losses [Fig. 19] and the output power is still sufficiently high above $70 \%$ of the imput power. We also notice that, while we have chosen relatively large $g$ the phenomenon is also observable (although less pronounced) for lower levels of the light absorption.

In practice, however, Figs 1 (a) and (b) will not correspond to a real experiment with an optical coupler, because in the standard settings its length, i.e. $Z$, is fixed. Instead, as we mentioned above, observation of the Zeno effects, can be achieved by varying wavelength of the light. From the panel (b) one concludes that the best observation of the phenomenon can be achieved at some intermediate lengths of the coupler, where, on the one hand, the power-like decay is already established and, on the other hand, the output power is still high so that the system is still in the nonlinear regime (we do not show transition to the linear regime, which for the data used in Fig. 1 occurs approximately at $Z \approx 0.3$. In our case the coupler lengths satisfying the above requirements correspond to the interval $0.2 \lesssim Z \lesssim 2$. Respectively, choosing $Z=2$ in the panels (c) and (d) we show how the output intensity depends on the wavelength of the incident beam, which can be manipulated experimentally. In, particular in panel (d) one clearly observes the linear Zeno effect as a dramatic increase of the output power (the transparency window of the coupler) exactly at the pick attenuation (see the dashed curve) achieved at the wavelength $\lambda_{0}$, as well as practically lossless propagation of the field in the nonlinear case (c.f. the solid line with dashed curves). Remarkably, for the strongly nonlinear case we also observe local increase of the output power, which however is preceded by the small decay of the power. The local decay of the intensity appears when the input power is approximately equal to the critical one $(\delta \approx 1)$.

So far, however, we have considered the case of zero phase mismatch between the two arms of the coupler. In fig. 2 we show the dependence on the phase mismatch between the two cores.

One observes that the input phase mismatch does not destroy the phenomenon, but can affect the output energy flow by the order of $10 \%$ (the relative energy distribution being practically unchanged).

\section{CONCLUSIONS}

To conclude, we have shown that by using a simple optical coupler subjected to the wavelength dependent absorption of the light in one of the arms, one can observe the linear and nonlinear Zeno effects. The phenomenon consists in increase of the output energy with increase of the absorption coefficient of one of the arms. The linear Zeno effect shows an especially strong dependence on the wavelength of the input signal, as this is expected from the design of the system. The nonlinear Zeno effect, observed at the intensities above the critical one, is characterized by a much larger transparency of the system, and consequently accompanied by much weaker dependence on the input wavelength.

It is interesting to mention that, recently, the effect of a light localization in linear coupler with the strong losses in one waveguide has been observed 26]. The authors attributed this phenomenon to the PT-symmetric configuration of their passive coupler (to which it can be reduced by the proper change of variables). Since the presence of the nonlinearity rules out the referred change of variables, the present work proposes an alternative explanation of the experiment reported in [26], and moreover, shows that this is a quite general phenomenon (non necessarily related to the PT-symmetry), which can be observed in linear and nonlinear systems and which open new possibilities for manipulating transmission of light by means of controllable absorption by making it either intensity of wavelength dependent.

\section{ACKNOWLEDGMENTS}

The authors greatly acknowledge stimulating discussions with Alex Yulin. FKA and VVK were supported by the 7th European Community Framework Programme under the grant PIIF-GA-2009-236099 (NOMATOS). VSS was supported by the CNPq of Brasil. 
[1] L. A. Khalfin, Dokl. Akad. Nauk SSSR 115, 277 (1957) [Sov. Phys. Dokl. 2, 232 (1958)]; Zh. Eksp. Teor. Fiz. 33, 1371 (1958) [Sov. Phys. JETP 6, 1053 (1958)].

[2] A. Degasperis, L. Fonda, and G. C. Ghirardi, Nuovo Cimento, 21, 471 (1974)

[3] L. Fonda, G. C. Ghirardi, and A. Rimini, Rep. Prog. Phys., 41, 587 (1978).

[4] L. A. Khalfin, Usp. Fiz. Nauk 160, 185 (1990).

[5] B. Misra and E. C. G. Sudarshan, J. Math. Phys. Sci. 18, 756 (1977).

[6] W. M. Itano, D. J. Heinzen, J. J. Bollinger, and D. J. Wineland, Phys. Rev. A 41, 2295 (1990)

[7] M. C. Fischer, B. Gutiérrez-Medina, and M. G. Raizen, Phys. Rev. Latt. 87, 040402 (2001)

[8] T. Nakanishi, K. Yamane, and M. Kitano, Phys. Rev. A 65, 013404 (2001)

[9] E. W. Streed, et. al., Phys. Rev. Lett. 97, 260402 (2006)

[10] N. Syassen et. al., Science 320, 1329 (2008)

[11] see e.g. P. Facchi, H. Nakazato, and S. Pascazio, Phys. Rev. Lett. 86, 2699 (2001); A.G. Kofman and G. Kurizki, Nature (London) 405, 546 (2000), Phys. Rev. Lett. 87, 270405 (2001); P. Facchi and S. Pascazio, Phys. Rev. Lett. 89, 080401 (2002); A. Barone, G. Kurizki, and A.G. Kofman, Phys. Rev. Lett. 92, 200403 (2004); I. E. Mazets, G. Kurizki, N. Katz, and N. Davidson, Phys. Rev. Lett. 94, 190403 (2005).

[12] P. Facchi and S. Pascazio, J. Phys. A: Math. Theor. 41, 493001 (2008).
[13] V. A. Brazhnyi, V. V. Konotop, V. M. Pérez-García, and H. Ott, Phys. Rev. Lett. 102, 144101 (2009)

[14] V. S. Shchesnovich, and V. V. Konotop, Phys. Rev. A 81, 053611 (2010).

[15] S. Longhi, Phys. Rev. Lett. 97, 110402 (2006).

[16] J. Rehacek, J. Perina, P. Facchi, S. Pascazio and L. Mista, Jr., Optics and Spectroscopy. 91, 501 (2001).

[17] Notice, that the optical analogy of the linear quantum Zeno effect described in [15] was based on existence of evanescent modes, i.e. on a different physical principle having and had very different physical manifestation.

[18] S. M. Jensen, IEEE J. Quantum Electron. 18, 1580 (1982).

[19] D. N. Christodoulides and R. I. Joseph, Opt. Lett. 13, 794 (1988)

[20] V.G. Taeed et al., Optics Express, 15, 9205 (2007).

[21] I.D. Chremmos, G. Kakarantas, and M. K. Usunoglu, Opt. Comm. 251, 339 (2005).

[22] Y. Ruan et al. Opt. Lett. 30, 2605 (2005).

[23] I.D. Aggarwal, J.C. Sanghera, and J. Optoelectr. Adv. Mat. 4, 665 (2002).

[24] J.S. Sanghera et al., J. Optoelectr. Adv. Mat. 8, 2148 (2006).

[25] E. M. Graefe, H. J. Korsch, and A. E. Niederle, Phys. Rev. Lett. 101, 150408 (2008).

[26] A. Guo et al. Phys. Rev. Lett. 103, 093902 (2009). 

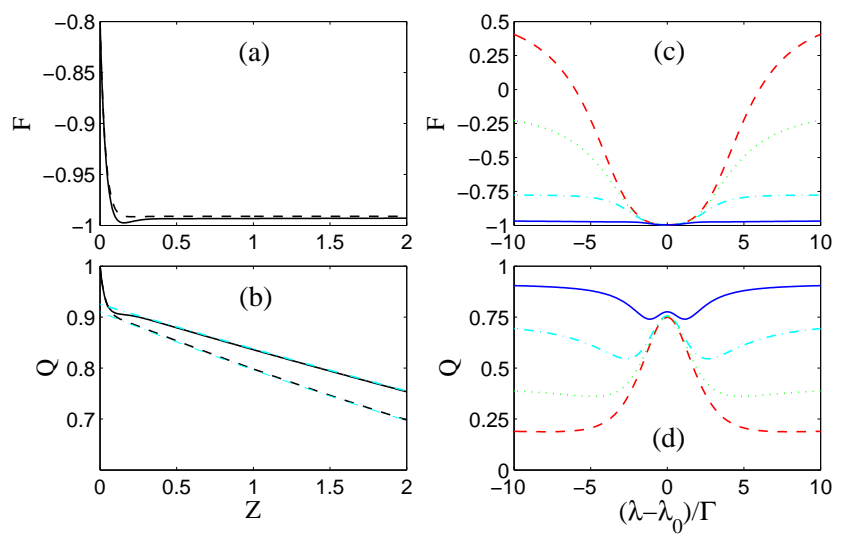

FIG. 1. (Color online) The relative (a), and the total (b) energy flows vs the propagation distance $Z$. Here $F(0)=$ $-0.8, \phi(0)=0, \beta_{2}=\beta_{1}=0, g=15, \delta=0$ (dashed lines) and $\delta=2.5$ (solid lines). The dash-dotted lines in panel (b) show the reduced rate attenuation given by Eq. (3) (also obtained by numerical simulations). In panels (c) and (d): the output energy distribution $(F$, panel (c)) and the total output energy flow $(Q$, panel (d)) in the coupler of the length $Z=2$, as functions of the deviation of the input light wavelength from the attenuation pick intensity $\lambda_{0}$. The output results for different values of $\delta, \delta=0$ (dashed), $\delta=0.5$ (dots), $\delta=1$ (dash-dot) and $\delta=2.5$ (solid), demonstrate the linear and nonlinear Zeno effects.
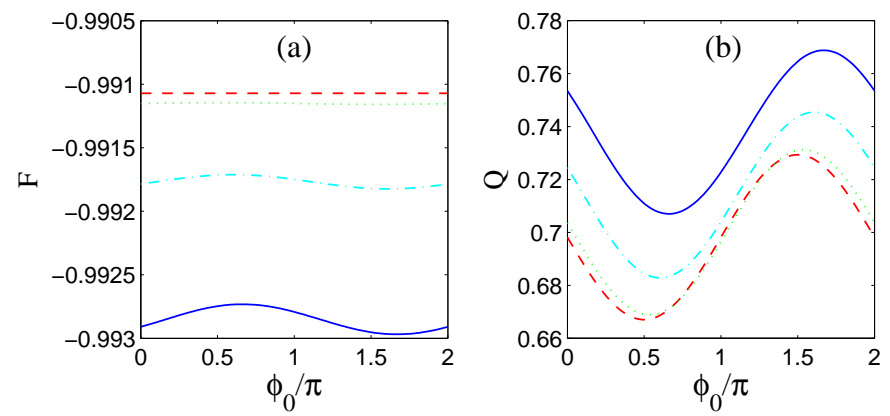

FIG. 2. (Color online) Output energy distribution ( $F$, panel (a)) and the total output energy $(Q$, panel (b)) vs the relative input phase $\phi(0)$. Here $F(0)=-0.8, g=15, Z=2$ and $\beta_{2}=\beta_{1}=0$. The lines correspond to different values of $\delta$, representing the nonlinear Zeno effect: $\delta=0$ (dashed), $\delta=0.5$ (dots), $\delta=1.5$ (dash-dot) and $\delta=2.5$ (solid). 